\title{
Dacriocistorrinostomia externa em crianças
}

\author{
Externaldacryocystorhinostomy in children
}

Fabio Henrique da Silva Ferraz ${ }^{1}$

Silvana Artioli Schellini ${ }^{2}$

Regina Hitomi Sakamoto ${ }^{1}$

Carlos Roberto Padovani ${ }^{3}$
Trabalho realizado no Departamento de Oftalmologia, Otorrinolaringologia e Cirurgia de Cabeça e Pescoço da Faculdade de Medicina de Botucatu - UNESP.

${ }^{1}$ Ex-residentes de Oftalmologia do Departamento de Oftalmologia, Otorrinolaringologia e Cirurgia de Cabeça e Pescoço da Faculdade de Medicina de Botucatu.

${ }^{2}$ Professor Adjunto do Departamento de Oftalmologia,

Otorrinolaringologia e Cirurgia de Cabeça e Pescoço

da Faculdade de Medicina de Botucatu.

${ }^{3}$ Professor Titular do Departamento de Bioestatística do Instituto de Biociências da Universidade Estadual Paulista - UNESP.

Endereço para correspondência: Silvana Artioli Schellini - Departamento de Oftalmologia, Otorrinolaringologia e Cirurgia de Cabeça e Pescoço da Faculdade de Medicina de Botucatu - Distr. Rubião Jr. s/n - Botucatu (SP) CEP 18600-000 - E-mail:sartioli@fmb.unesp.br Recebido para análise em 07.08.2002

Versão revisada recebida em 06.01.2003

Aprovação em 07.04.2003

Nota Editorial: Pela análise deste trabalho e por sua anuência na divulgação desta nota, agradecemos aos Drs. Suzana Matayoshi e Waldir Martins Portellinha.

\section{RESUMO}

Objetivo: Avaliar a eficácia da dacriocistorrinostomia(DCR) externa realizada em crianças. Métodos: O estudo foi retrospectivo, avaliando-se 16 crianças com idade inferior a 10 anos, portadoras de obstrução da via lacrimal e submetidas a DCR externa, no período de 1986 até 2001. Foram avaliadas as características dos portadores, as queixas apresentadas, o exame e o tipo de cirurgia efetuada, assim como o resultado do tratamento. Resultado: A amostra estudada não diferiu quanto ao sexo e faixa etária. A cirurgia mais realizada foi DCR sem o uso de entubação de vias lacrimais (87,5\%). 62,5\% tiveram resolução da epífora com um único procedimento e 81,0\%, com dois. Conclusão: O resultado da DCR externa, quando realizada em crianças, é semelhante ao observado em adultos.

Descritores: Dacriocistorrinostomia; Aparelho lacrimal/cirurgia; Estudo retrospectivo; Criança

\section{INTRODUCÃO}

A epífora pode ocorrer em crianças, quando, em grande parte, decorre de obstrução do ducto nasolacrimal devido à persistência da membrana de Hasner ou estenose do sistema de drenagem lacrimal abaixo do saco lacrimal $^{(1-2)}$.

O quadro clínico de epífora e secreção pode ter resolução espontânea na grande maioria dos portadores ${ }^{(1)}$. Quando isso não ocorre, massagens e/ou antibioticoterapia tópica podem ser úteis.

No entanto, em poucos casos há persistência dos sintomas e aparecimento de quadros infecciosos, tornando necessário utilizar tratamentos intervencionistas, como a sondagem das vias lacrimais, a entubação, a dilatação intracanalicular usando cateter balão e a dacriocistorrinostomia $(\mathrm{DCR})^{(3-4)}$.

Quando a sondagem e a entubação não apresentam resultados satisfatórios ou quando há dacriocistite aguda, a DCR pode ser realizada pelas vias externa ou endonasal ${ }^{(2,5)}$.

No entanto, poucos são os relatos enfocando a resolutibilidade da cirurgia quando realizada em crianças.

Assim, o objetivo deste estudo é avaliar a eficácia da DCR externa, realizada como tratamento da obstrução de vias lacrimais, em crianças abaixo de 10 anos.

\section{MÉTODOS}

Foram avaliadas retrospectivamente 16 crianças (16 vias lacrimais) com idade inferior a 10 anos, portadoras de obstrução das vias lacrimais e submetidas a DCR externa no Hospital das Clínicas de Botucatu/UNESP, entre os anos de 1986 e 2001. 
O diagnóstico da obstrução foi realizado através dos achados clínicos (epífora, secreção espontânea ou à expressão e teste de Milder positivo) e/ou radiográficos, avaliando-se a presença de obstrução ou dilatação, após a injeção de contraste oleoso nas vias lacrimais.

Dez crianças $(62,5 \%)$ haviam sido sondadas anteriormente e 1 criança $(6,25 \%)$ havia sido submetida a entubação como primeiro procedimento, não tendo havido resolução dos sintomas. Outras 5 crianças tiveram a cirurgia indicada devido à presença de dilatação importante do saco lacrimal no exame dacriocistográfico.

Estabeleceu-se a idade da queixa e a época em que se procedeu a cirurgia.

As crianças foram submetidas à correção cirúrgica através da DCR externa. Apenas 2 pacientes foram submetidos à DCR associada a entubação com silicone, devido à lesão da mucosa nasal durante o ato cirúrgico. A técnica da DCR externa utilizada foi com abertura retilínea da pele e tecido subcutâneo sobre o dorso nasal, exposição do saco lacrimal, abertura da janela óssea com osteótomo de Citelli modificado na altura do meato nasal médio, abertura da mucosa nasal e do saco lacrimal em formato de $\mathrm{C}$, com ressecção do folheto posterior e sutura do anterior. Todas as cirurgias foram realizadas por cirurgiões em formação.

Os pacientes foram acompanhados no pós-operatório em período que variou de 6 meses a 2 anos.

O resultado da cirurgia foi avaliado de acordo com a remissão ou persistência dos sintomas de epífora ou secreção no pós-operatório.

Os casos que apresentaram recorrência da obstrução foram submetidos a novo tratamento cirúrgico com as técnicas descritas acima, tendo-se realizado 3 DCR, 1 DCR com entubação com silicone e 1 conjuntivorrinostomia com colocação de tubo de Jones, empregando-se novamente os critérios de cura no pós-operatório.

Os dados obtidos foram submetidos à análise estatística usando o teste do Qui-quadrado.

\section{RESULTADOS}

O grupo estudado apresentou-se bastante homogêneo quanto à idade, não havendo predomínio entre as classes $(\mathrm{P}>0,05)$. Das 16 crianças, 9 eram do sexo feminino, não havendo predomínio significativo entre os sexos $(\mathrm{P}>0,05)$.

A maioria dos pacientes $(87,5 \%)$ manifestou os primeiros sintomas da obstrução precocemente, isto é, até os 6 meses de idade, sendo que apenas $2(12,5 \%)$ apresentaram a queixa inicial após esta idade $(\mathrm{P}<0,0001)$.

A dacriocistografia revelou obstrução em 100,0\% das vias lacrimais examinadas. Foi identificada obstrução no nível da membrana de Hasner em 10 pacientes (63,5\%), no seio de Arlt em 5 (31,2\%) e no canalículo comum em 1 (6,2\%).

A idade predominante das crianças na época da cirurgia esteve entre 1 a 5 anos e 5 a 10 anos, em igual proporção.

O procedimento cirúrgico adotado na maioria dos casos foi a DCR externa isolada (87,5\%), seguido da DCR com entuba- ção $(\mathrm{P}<0,0001)$. A entubação associada a DCR foi utilizada em dois pacientes em que houve lesão da mucosa nasal durante o procedimento cirúrgico.

Houve remissão dos sintomas em $62,5 \%$ dos pacientes com apenas um procedimento cirúrgico. Cinco crianças necessitaram de uma segunda intervenção devido a insucesso da DCR. As causas do insucesso foram: a fibrose no local da ponte mucosa com fechamento da fístula em 2 pacientes, falha no funcionamento da fístula associada a novos episódios de dacriocistite aguda em 2 pacientes e fistulização para a pele em um paciente.

Após a reoperação, apenas 2 casos (12,5\%) persistiram com a epífora.

\section{DISCUSSÃO}

A obstrução das vias lacrimais pode ser congênita ou adquirida, primária ou secundária a diversas causas, como traumatismo e inflamação. A congênita, em 90,0\% dos casos, decorre da persistência da membrana de Hasner ou estenose do ducto nasolacrimal ${ }^{(6)}$.

O tratamento da obstrução nasolacrimal congênita pode ser conservador, utilizando-se de massagens, com bons resultados, principalmente em crianças abaixo de 1 ano de idade, onde o índice de sucesso chega a 90,0\%( ${ }^{(1)}$.

Quando o tratamento conservador falha, ou existem sinais de infecção aguda, pode-se necessitar do tratamento intervencionista, sendo a sondagem, o primeiro deles, com índice de cura de 70,0 a $86,0 \%{ }^{(7-9)}$. A época ideal para a sondagem é discutível, recomendando-se condutas conservadoras pelo menos até o $6^{\circ}$ mês, com sondagem após, caso persistam os sintomas ${ }^{(10)}$. A entubação é a segunda opção de tratamento, sendo sua eficácia melhorada quando associada com a endoscopia nasal ${ }^{(1)}$.

Quando a sondagem e a entubação não são bem sucedidas, torna-se necessária a realização de procedimentos mais invasivos, como a DCR.

Estudos apontam a etiologia inflamatória da obstrução como sendo o principal fator para a indicação da $\mathrm{DCR}^{(11)}$. Assim, a DCR é indicada, sempre que possível, em crianças acima de 1 ano de idade e que apresentam dacriocistite recorrente, com índices de cura de 90,0 a $95,0 \%{ }^{(4)}$.

No presente estudo, a cirurgia foi realizada em crianças entre 1 e 10 anos de idade. Porém, se crianças abaixo de 1 ano apresentam saco lacrimal dilatado e/ou dacriocistite aguda, pode ser necessária a intervenção mais precocemente.

A amostra aqui estudada apresentou remissão dos sintomas após um único procedimento em $62,5 \%$ dos pacientes. Houve necessidade de reoperação em 5 crianças e, após o segundo tratamento, o índice elevou-se para 81,0\%.

Estudos avaliando a DCR externa em adultos mostram melhora dos sintomas em cerca de $90,0 \%$ dos pacientes ${ }^{(12-13)}$. Valores semelhantes são apontados quando se trata de DCR endoscópica $^{(14-15)}$. No entanto, alguns trabalhos apresentam índices de sucesso menores com a DCR endoscópica (75,0\%), quando comparados com a DCR externa ${ }^{(16-17)}$. O resultado é ainda pior quando se realiza a conjuntivorrinostomia $(67,9 \%)$, devido à possibilidade de perda ou deslocamento do tubo de Jones ${ }^{(18)}$. 
Em crianças, poucos são os estudos sobre a DCR externa, sendo que a maioria dos comentários existente, versa sobre a DCR endoscópica, cujos índices de sucesso são controversos, variando de cura em $90,0 \%{ }^{(6,19)}$ a $100,0 \%$ (avaliação de apenas 4 crianças) ${ }^{(5)}$ até falha em $100,0 \%$, desaconselhando-se esta técnica para pacientes pediátricos ${ }^{(2)}$. Portanto, os poucos estudos que existem sobre o assunto, o abordam de maneira unilateral, versando sobre a DCR endoscópica, e são controversos.

Porém, mais que a técnica empregada, o resultado é influenciado pela experiência do cirurgião, sendo relatados com a DCR externa, altos índices de cura em crianças ${ }^{(20)}$. É necessário lembrar que os cirurgiões no presente estudo eram residentes de Oftalmologia, o que pode ter influenciado os índices de sucesso cirúrgico.

Comparando-se os resultados obtidos em crianças com os observados em adultos no mesmo serviço ${ }^{(21)}$, observa-se que os índices de sucesso são semelhantes na população adulta e infantil.

As dimensões reduzidas das estruturas anatômicas fazem com que seja mais difícil realizar a cirurgia em crianças. Outras diferenças existentes são a necessidade de realização de todas as cirurgias sob anestesia geral e a dificuldade do exame da criança no pós-operatório.

A reação inflamatória intensa, assim como a tendência de crescimento acentuado de tecido cicatricial que ocorre em crianças $^{(22)}$, poderia ser um fator determinante no resultado final da DCR, com possibilidade de fechamento da fístula cirúrgica. No entanto, esta hipótese não se confirmou, uma vez que os resultados desfavoráveis não ocorreram por excesso de inflamação.

Uma das causas da falha na DCR é a presença de lesão canalicular, sendo necessária a correção microcirúrgica do canalículo $^{(23)}$. Um dos pacientes estudados apresentou este problema, sendo submetido à colocação do tubo de Jones para a correção da obstrução canalicular. No entanto, não obteve boa evolução, devido ao deslocamento do tubo, complicação comum com a utilização deste procedimento.

Nas reoperações, duas crianças necessitaram de DCR com entubação, sendo que uma evoluiu para cura e a outra apresentou recorrência do quadro. A entubação com tubo de silicone deixado por longo período apresenta melhores resultados, mesmo quando há obstrução canalicular ou agenesia de ducto nasolacrimal ${ }^{(24)}$.

Os resultados obtidos no presente estudo mostram que a DCR quando realizada em crianças possui comportamento semelhante ao observado em adultos.

\section{ABSTRACT}

Purpose: To evaluate external dacryocystorhinostomy (DCR) performed in children. Methods: Retrospective study of 16 children under 10 years old, with lacrimal obstruction and treated with the external DCR procedure, between 1986 and 2001. The patient's features, complaints, examinations, utilized surgical technique and its results were evaluated. Results: The sample showed no differences regarding sex and age. $87.5 \%$ of the patients were submitted to external DCR without intubation. $62.5 \%$ had resolution of their epiphora with only one surgery and $81.0 \%$, with one reoperation. Conclusion: External DCR, performed, in childhood, has a similar behavior to that observed in adults.

Keywords: Dacryocistorhinostomy; Lacrimal apparatus/surgery; Retrospective studies; Child

\section{REFERÊNCIAS}

1. MacEwen CJ, Young JD, Barras CW, Ram B, White PS. Value of nasal endoscopy and probing in the diagnosis and management of children with congenital epiphora. Br J Ophthalmol 2001;85:314-8.

2. Doyle A, Russell J, O’Keefe M. Paediatric laser DCR. Acta Ophthalmol Scand 2000;78:204-5.

3. Tahat AA. Dacryostenosis in newborns: probing, or syringing, or both? Eur J Ophthalmol 2000;10:128-31.

4. Struck HG, Weidlich R. Indications and prognosis of dacryocystorhinostomy in childhood. A clinical study 1970 - 2000. Ophthalmologe 2001;98:560-3.

5. Cunningham MJ, Woog JJ. Endonasal endoscopic dacryocystorhinostomy in children. Arch Otolaryngol Head Neck Surg 1998;124:328-33.

6. Piane R, Romano L, Nuti D, Romano F, Passàli D. Endonasal dacryocystorhinostomy: a personal experience. Acta Otorhinolaryngol Ital 1999;19:255-9.

7. Chiesi C, Guerra R, Longanesi L, Fornaciari M, Morano RP. Congenital nasolacrimal duct obstruction: therapeutic management. J Pediatr Ophthalmol Strabismus 1999;36:326-30.

8. Kushner BJ. The management of nasolacrimal duct obstruction in children between 18 months and 4 years old. J AAPOS 1998;2:57-60.

9. Goldblum TA, Summers CG, Egbert JE, Letson RD. Office probing for congenital nasolacrimal duct obstruction: a study of parental satisfaction. J Pediatr Ophthalmol Strabismus 1996;33:244-7.

10. Da Pozzo S, Pensiero S, Perissutti P. Management of congenital nasolacrimal duct obstruction. Timing of probing. Minerva Pediatr 1995;47:209-13.

11. Faria, JL. Dacriocistorrinostomia intranasal: contribuição ao tratamento cirúrgico [tese]. Curitiba: Universidade Federal do Paraná; 1997.

12. Yung MW, Hardman-Lea S. Analysis of the results of surgical endoscopic dacryocystorhinostomy: effect of the level of obstruction. Br J Ophthalmol 2002;86:792-4.

13. Delaney YM, Khooshabeh R. External dacryocystorhinostomy for the treatment of acquired partial nasolacrimal obstruction in adults. Br J Ophthalmol 2002;86:533-5.

14. Wormald PJ. Powered endoscopic dacryocystorhinostomy. Laryngoscope 2002;112:69-72.

15. Iturralde PPV, Reis FO, Oliveira RA, Cahali M, Voegels RL. Resultados da técnica cirúrgica endoscópica endonasal para dacriocistorrinostomia. Rev. bras. Otorrinolaringol 2001;67:527-9.

16. Woog JJ, Kennedy RH, Custer PL, Kaltreider SA, Meyer DR, Camara JG. Endonasal dacryocystorhinostomy: a report by the American Academy of Ophthalmology. Ophthalmology 2001;108:2369-77.

17. Ibrahim HA, Batterbury M, Banhegyi G, McGalliard J. Endonasal laser dacryocystorhinostomy and external dacryocystorhinostomy outcome profile in a general ophthalmic service unit: a comparative retrospective study. Ophthalmic Surg Lasers 2001;32:220-7.

18. Hannas TR, Soares EJ, França VP, Figueiredo ARP, Bedran EGM. Problemas associados com a conjuntivo-dacriocistorrionostomia. Rev. bras. Oftalmol 1995;54:57-61.

19. Vanderveen DK, Jones DT, Tan H, Petersen RA. Endoscopic dacryocystorhinostomy in children. J AAPOS 2001;5:143-7.

20. Barnes EA, Abou-Rayyah Y, Rose GE. Pediatric dacryocystorhinostomy for nasolacrimal duct obstruction. Ophthalmology 2001;108:1562-4.

21. Schellini AS, Itoda L, Silva MRBM. Dacrioestenose: análise dos casos atendidos em nosso serviço. Rev Bras Oftalmol 1990;49:43-8.

22. Nowinski TS, Flanagan JC, Mauriello J. Pediatric dacryocystorhinostomy. Arch Ophthalmol 1985;103:1226-8

23. Struck HG, Tost F. Postoperative complications of Toti DCR (dacryocystorhinostomy). An indication for canalicular surgery. Ophthalmologe 1999;96:443-7.

24. Häusler R, Caversaccio M. Microsurgical endonasal dacryocystorhinostomy with long-term insertion of bicanalicular silicone tubes. Arch Otolaryngol Head Neck Surg 1998;124:188-91. 